\title{
LA INSTRUMENTACIÓN EM ESPAÑA DE LAS POLÍTICAS EUROPEAS DE EMPRENDIMENTO ¿CREACIÓN DE EMPLEO O PROFUNDIZACIÓN DE LA CRISIS DEL EMPLEO ASALARIADO? ${ }^{12}$
}

\author{
Laureano Martinez ${ }^{3}$ \\ Victoria Bogino Larrambebere ${ }^{4}$
}

\section{Resumen}

En el siguiente artículo se propone un análisis de la instrumentación en España de las políticas de promoción del emprendimiento propuestas por las instituciones europeas. Dicho análisis se basa en la perspectiva teórica y metodológica de los Instrumentos de Acción Pública (IAP). Inicialmente, se exponen los criterios teóricos y metodológicos mencionados, para posteriormente proceder a analizar la forma en que el Estado español ha instrumentado los planes propuestos por la Comisión Europea, indagando sobre los efectos de dicha instrumentación en el régimen de empleo. Se concluye en que los instrumentos sugeridos por las instituciones europeas y adoptados por España conducen a un tipo de emprendedor de tipo individual y por necesidad, que queda fuera de los marcos establecidos por el derecho del trabajo.

Palabras-clave: emprendedores, políticas sociales europeas, empleo asalariado, instrumentos de acción pública.

\section{Resumo}

O seguinte artigo propõe uma analise da instrumentação das Políticas de promoção do empreendimento proposto pelas instituições europeias na Espanha. Esta analise baseia-se na perspectiva teórica e metodológica dos Instrumentos de Ação Publica (IAP). Inicialmente, expõemse os critérios teóricos e metodológicos mencionados, para posteriormente analisar a forma em que o estado espanhol tem instrumentado os planos proposto pela Comissão Europeia, indagando os efeitos da dita instrumentação no regime de trabalho. Conclui-se que o modo de instrumento sugerido pelas instituições europeias e adotado pela Espanha, conduz a um tipo de empreendedor do tipo individual e por necessidade, que fica fora dos marcos estabelecido pelo direito do trabalho.

Palavras-chave: Empreendedores, políticas sociais europeias, trabalho assalariado, instrumentos de Ação Publica.

\footnotetext{
Abstract

The aim of this paper is to analyze the implementation of entrepreneurship policies in Spain proposed by the European institutions. This analysis is based on the theoretical and methodological perspective of Public Action Instruments (IAP). Initially, we outline the aforementioned approach, in order to subsequently analyze how the Spanish State has implemented the recommendations proposed by the European Commission, inquiring about the effects of such instrument in the regime of employment. We conclude that the type of instruments suggested by the European institutions and adopted by Spain leads to a kind of individual entrepreneurship, which is outside the framework of the labor law. Keywords: entrepreneurship, European social policies, wage-earning labour, Public Action Instruments.

${ }^{1}$ DOI deste artigo: $10.5380 /$ recp.v6i1.37493.

${ }^{2}$ Este artículo se inscribe en el marco de la investigación predoctoral llevada a cabo por los autores. Una versión preliminar del mismo fue presentada en el Congreso CABISE'14 Revaluando el Estado de Bienestar en el sur de Europa, 5 y 6 de junio de 2014, Oviedo, España. Los autores agradecen a los evaluadores de la revista por los comentarios recibidos sobre la primera versión de este escrito.

${ }^{3}$ Candidato a Doctor por la Facultad de Ciencias Humanas y Sociales de la Universidad Pública de Navarra, Espanã. E-mail: martinez.laureano@gmail.com

${ }^{4}$ Candidata a Doctora em Sociología. Universidad Autónoma de Barcelona. E-mail: victoriabogino@gmail.com
} 


\section{INTRODUCCIÓN}

En este artículo se propone un análisis de la instrumentación de políticas sociales y de empleo europeas en España. Con ello, se pretende cumplir un doble objetivo. Por un lado, contribuir a la difusión, en el marco de investigaciones en Ciencia Política, de la perspectiva de los Instrumentos de Acción Pública (IAP) como metodología de análisis (HALPERN et al., 2014; LASCOUMES y LE GALÈS, 2005; LASCOUMES Y SIMARD, 2011). Por otro, llevar a cabo un análisis de caso: la instrumentación en España de las políticas europeas de promoción del emprendimiento y el espíritu de empresa.

Desde la Estrategia Europea por el Empleo de 1997, primer intento de coordinación de políticas de empleo a nivel comunitario, las instituciones europeas han promovido el "espíritu de empresa" y el emprendimiento como medidas de creación de empleo y crecimiento económico. Para llevar a cabo nuestro objetivo, nos centraremos en la forma en que se han instrumentado esas medidas en España desde el año 2010, en el marco de la Estrategia Europa 2020. Para ello, planteamos los siguientes interrogantes: ¿cómo se instrumentan las políticas europeas de promoción del emprendimiento en España? ¿Cuáles son sus efectos en el régimen de empleo?

Para responder a estos interrogantes, el artículo se divide en tres apartados. En primer lugar, se exponen los lineamientos teóricos y metodológicos del análisis de los Instrumentos de Acción Pública. Posteriormente, a partir de esos lineamientos, se abordan los planes y recomendaciones emitidos por la Comisión Europea sobre promoción del emprendimiento y el espíritu de empresa. Por último, se analiza la instrumentación que el Estado español ha realizado de dichas recomendaciones. Se concluye sobre ciertas implicancias de esta instrumentación en la regulación del régimen de empleo.

\section{LAS POLÍticAS PÚBLICAS DESDE LA PERSPECTIVA DE LOS INSTRUMENTOS DE ACCIÓN PÚBLICA}

Como sub-campo del análisis de políticas públicas, la perspectiva de los instrumentos de la acción pública aborda la forma en que se operacionaliza la racionalidad gubernamental (LABORIER y LASCOUMES, 2005), es decir, la forma en que se establecen para el ejercicio de gobierno determinados fines, estrategias, mecanismos y un campo de intervención (BRUNO, 2006). Más específicamente, se trata de analizar el conjunto de problemas vinculados a la elección y la utilización de ciertas herramientas, técnicas o dispositivos que permiten materializar y operacionalizar los programas de la acción gubernamental. El análisis de la elección de determinados instrumentos permite poner de relieve la intencionalidad 
política de su elección, en relación a la orientación de conductas individuales y colectivas (LASCOUMES y SIMARD, 2011).

Si partimos de considerar de modo general que la acción pública es un conjunto heterogéneo de prácticas construidas por técnicas, normas, objetivos, recursos y actores, la noción de instrumento de acción pública permite ir más allá de las perspectivas funcionalistas, que se interesan principalmente por los objetivos de las políticas públicas y por analizarla como "solución a problemas", para observar la acción pública a partir del ángulo de los mecanismos que estructuran los programas de gobierno. En este sentido, un instrumento de acción pública

\begin{abstract}
[...] puede ser definido como un dispositivo técnico, de vocación genérica, portador de una concepción concreta de la relación política/sociedad y sostenido por una concepción de la regulación. Esta instrumentación adquiere clásicamente las formas de directivas más o menos sancionadas (leyes, reglamentos), de relaciones financieras (deducciones fiscales, ayudas económicas directas e indirectas) y de conocimiento y comparación de poblaciones (mediciones estadísticas) [...] Se trata de no pensar el Estado por lo alto sino de colocar el foco sobre la acción pública a partir de los dispositivos y de las prácticas [concretas].... (LABORIER y LASCOUMES, 2005: 61).
\end{abstract}

Además, los instrumentos son indisociables de los agentes sociales que lo ponen en juego, por lo que no son reducibles a una racionalidad técnica pura, sino que responden fundamentalmente a una estrategia política. Su "vocación genérica" indica que se aplica a problemas sectoriales diversos y que son movilizados por diferentes políticas en su forma y su fundamento. Por ello, los autores que sostienen esta perspectiva remarcan que el problema no es la "naturaleza" del instrumento, sino centrarse en los "efectos" que generan: los efectos generados por los instrumentos en su "autonomía relativa" y los efectos políticos y las relaciones de poder en que se inscriben (LASCOUMES y SIMARD, 2011).

En el siguiente cuadro se resumen los principales instrumentos de acción pública propuestos por LASCOUMES y SIMARD (2011).

Cuadro 1 - Instrumentos de acción pública.

\begin{tabular}{|c|c|c|}
\hline Tipo de instrumento & Tipo de vínculo político & Tipo de legitimidad \\
\hline Legislativo y reglamentario & Estado regulador de lo social & $\begin{array}{c}\text { Imposición de un interés general } \\
\text { por los representantes electos o } \\
\text { altos cargos gubernamentales. }\end{array}$ \\
\hline Económico y fiscal & $\begin{array}{c}\text { Estado productor de riqueza / } \\
\text { Estado redistribuidor }\end{array}$ & $\begin{array}{c}\text { Búsqueda de una utilidad colectiva. } \\
\text { Búsqueda de eficacia económica y } \\
\text { social. }\end{array}$ \\
\hline Convencional e incitativo. & Estado movilizador & $\begin{array}{c}\text { Búsqueda de compromisos } / \\
\text { direccionar acciones. }\end{array}$ \\
\hline Informativo y comunicacional & $\begin{array}{c}\text { Espacio público } \\
\text { democratizado }\end{array}$ & $\begin{array}{c}\text { Explicitación de las decisiones y } \\
\text { responsabilización de los actores. }\end{array}$ \\
\hline
\end{tabular}




\begin{tabular}{|c|c|c|}
\hline $\begin{array}{c}\text { Normas y estándares de } \\
\text { "Buenas prácticas". }\end{array}$ & Mecanismos de competencia. & $\begin{array}{c}\text { Mixto: científico-técnico y } \\
\text { democráticamente negociada o } \\
\text { competencia por mecanismos de } \\
\text { mercado. }\end{array}$ \\
\hline
\end{tabular}

Fuente: Lascoumes y Simard (2011).

Cabe destacar que las políticas no son de carácter "monoinstrumental", sino que combinan diferentes instrumentos. Por ello, desde el punto de vista metodológico, la perspectiva de los IAP requiere examinar las modalidades concretas de instrumentación de una política. En ese sentido, tomar las políticas de promoción del emprendimiento como objeto de estudio desde la perspectiva de los instrumentos, implica abordar un conjunto diverso de elementos, como documentos oficiales (comunicaciones, Planes Nacionales de Reforma, directrices, leyes, programas), indicadores estadísticos, medidas económicas y fiscales, presupuestos teóricos y argumentos políticos. Nuestra interrogación a las políticas de emprendimiento se dirige a su dimensión normativa o programática, es decir, en tanto instrumento que establece criterios de acción y modos de comportamiento, y que es creador de determinados vínculos sociales en el mundo de las relaciones laborales. De este modo, se intentará reconstruir el régimen de prácticas que anclan el instrumento en un espacio concreto.

A su vez, como hemos mencionado, interrogarse por la acción pública desde su instrumentación requiere problematizar los efectos que conlleva la elección de un determinado instrumento (LASCOUMES y LE GALÈS, 2005). Estos efectos no deben ser entendidos de forma determinista sino de modo probabilístico: "los instrumentos [...] son susceptibles de tener ciertos efectos, pero los usos que se hacen de ellos y la resistencia hacia los mismos pueden cambiar completamente la situación y transformarlos" (HALPERN, et al. 2014: 39). En este sentido, el problema de investigación que sustenta este artículo hace referencia a los efectos que en el mundo del trabajo presenta el emprendedorismo como componente del régimen de empleo, frente a la norma del empleo asalariado (PRIETO, 2013). Como veremos en distintos ejemplos, tanto en los documentos oficiales de todos los niveles gubernamentales como en la legislación, se encuentran referencias explícitas al trabajo asalariado entendido como contracara del emprendedor. De allí que nos interese problematizar la forma en que la instrumentación de las recomendaciones europeas en el Estado español afecta el régimen de empleo, en un contexto de crisis del trabajo asalariado (ALONSO, 2007). 


\section{LAS POLÍTICAS DE EMPRENDIMENTO EUROPEAS}

\subsection{El método abierto de coordinación como "macro-instrumento" del gobierno del empleo}

La perspectiva de los IAP resulta pertinente para abordar las políticas sociales y de empleo europeas por, al menos, el siguiente motivo. Este tipo de políticas, en su nivel supranacional, se limitan a recomendaciones, directrices o Planes de acción de carácter general y no obligatorio para los estados miembros. Es decir, las políticas sociales y de empleo son competencias fundamentales de los Estados, y el papel de la Unión es el de proponer y coordinar los lineamientos generales que luego los estados implementan.

En el tránsito entre distintas instancias gubernamentales, la instrumentación de las políticas adopta una 'fisonomía' que corresponde a las particularidades de cada país y, eventualmente, de cada región que lo integra, como en el caso de las Comunidades Autónomas españolas. Para analizar este tipo de regulación supranacional, Dehousse (2004) propone hablar del "macroinstrumento" que la conforma. El Método Abierto de Coordinación (MAC) es el mecanismo mediante el cual se hacen efectivas las competencias comunitarias de coordinación. Es el proceso por el cual los Estados miembros acuerdan objetivos comunes, preparan sus Programas Nacionales de Reforma y evalúan la acción de otros estados a partir del mecanismo de "buenas prácticas" o benchmarking (BRUNO, 2006). Si bien los antecedentes del MAC ya figuraban en la primera Estrategia Europea para el Empleo de 1997, fue en el Consejo de Lisboa de 2000 cuando se lo definió como un "planteamiento totalmente descentralizado de acuerdo con el principio de subsidiariedad, en el cual la Unión, los Estados miembros, los niveles regionales y locales, así como los interlocutores sociales y la sociedad civil, participan activamente, mediante distintas formas de colaboración” (CONSEJO EUROPEO, 2000). Según se establece en las conclusiones del Consejo, este método se basa en las siguientes cuatro fases de aplicación: 1) establecimiento de directrices; 2) fijación de indicadores cuantitativos y cualitativos basados en las "buenas prácticas", de modo de poder establecer criterios de comparación y evaluación; 3) plasmar las directrices a nivel de los Estados miembro, según las características de cada país; y 4) proceder a un seguimiento y evaluación de las políticas recomendadas. 
A partir del MAC se establecerá entonces un particular modo de gobierno, llamado gobernanza europea (COMISIÓN EUROPEA, 2001), de carácter supranacional, basado en toda una gramática novedosa en materia de cooperación interestatal (BRUNO, 2006). Representa, en suma, un tipo de instrumento caracterizado por su carácter flexible, por lo que suele inscribirse en el ámbito del "soft law" (DEHOUSSE, 2003). Del Método Abierto de Coordinación destacaremos un punto de particular interés para nuestro trabajo, como son los Programas Nacionales de Reforma. Es allí donde se plasman los acuerdos entre cada Estado y la Unión, en función a la situación de cada Estado, bajo un criterio de correspondencia entre las política macroeconómicas, microeconómicas y de empleo.

En el caso que nos ocupa, desde el comienzo de la crisis económica en el año 2007, la Comisión y el Consejo recomiendan a España emprender numerosas reformas, con la finalidad de recuperar el crecimiento económico y generar empleo. En ese marco, una de las medidas en que más han insistido las instituciones europeas ha sido en las políticas de promoción del emprendimiento. España se ha comprometido en sus Programas Nacionales de Reformas de 2010 a 2013 a impulsar estas medidas y posteriormente las ha efectuado siguiendo al pie dela letra las recomendaciones sugeridas desde Bruselas. Por citar un ejemplo, en la Ley 11/2013, del 26 de julio, de medidas de apoyo a los emprendedores y de estímulo del crecimiento y de la creación de empleo, se establece que: "la presente ley [...], responde a las recomendaciones que, en materia de empleo joven, ha realizado la Comisión Europea y se enmarca dentro del Plan Nacional de Reformas puesto en marcha por el Gobierno" (BOE, 2013a).

\subsection{El emprendimiento en el marco de las estratégias europeas de desarrollo}

Existen a nivel europeo tres estrategias coordinadas en materia de crecimiento y empleo. La primera, llamada Estrategia Europea por el Empleo (EEE), fue lanzada en el marco de la Cumbre Europea de Luxemburgo en 1997. Posteriormente, en el año 2000, la Estrategia de Lisboa propuso un amplio conjunto de medidas orientadas a tres ejes: económico, social y medioambiental. Por último, en 2010, inmersa la región en una profunda crisis económica y social, se estableció la Estrategia Europa 2020. Allí, en base a la evaluación de las estrategias anteriores, se establecen los objetivos y medidas en materia de crecimiento y empleo de la Unión para el año 2020, bajo la consigna "Una Estrategia para un crecimiento inteligente, sostenible e integrador". 
El desarrollo de las mencionadas estrategias no ha sido lineal y ha experimentado diversas modificaciones. Si bien es cierto que en términos generales han procurado integrar los criterios centrales plasmados en aquella primera Estrategia Europea para el Empleo, han sido objeto de diversos desplazamientos y han reorientado los objetivos e instrumentos planteados inicialmente. Independientemente de las modificaciones que introdujo cada una de estas estrategias, las políticas de emprendimiento han sido uno de los pilares desde su inicio hasta la actualidad. A lo largo de su desarrollo, estas políticas fueron adquiriendo una especificidad propia, que quedó plasmada con claridad en el último plan de la Comisión, denominado Plan de acción emprendedorismo 2020. Relanzar el espíritu emprendedor en Europa, del año 2013 (COMISIÓN EUROPEA, 2013).

Inicialmente, en el marco de la EEE, la Comisión Europea publicó en 1998 una comunicación al Consejo titulada Fomento del espíritu de empresa en Europa: prioridades para el futuro. De este modo comenzaban a coordinarse las medidas de apoyo al emprendedorismo y el espíritu de empresa. Estas primeras recomendaciones de medidas sentaron las bases de las posteriores políticas de promoción del emprendedorismo, para lo cual la Comisión proponía una doble estrategia: por un lado, medidas para que las personas se animen a hacerse empresarios y dotarlas de la cualificación necesaria para ello, lo que entraña "reformas en los sistemas de educación y formación y cambios culturales" (COMISIÓN EUROPEA, 1998: 3). Por otro, se propone la creación de un entorno económico favorable a la generación, el crecimiento y la transmisión de empresas, a través de "una radical simplificación administrativa, la mejora del entorno normativo y financiero, y el acceso a programas comunitarios como los de I+D y los Fondos Estructurales” (Ibídem).

Posteriormente, ya en el marco de la -inicialmente optimista- Estrategia de Lisboa, esas recomendaciones se plasmaron en el Plan de acción denominado El programa europeo en favor del espíritu empresarial (COMISIÓN EUROPEA, 2004). Allí se establecen cinco ámbitos políticos estratégicos para los fines del Plan: 1) Fomentar la mentalidad empresarial, 2) Animar a un mayor número de personas a convertirse en empresarios, 3) Orientar a los empresarios hacia el crecimiento y la competitividad; 4) Mejorar el flujo de financiación; 5) Crear un entorno administrativo y reglamentario más favorable para las PYME. 
Finalmente, ya en el marco de la Estrategia Europa 2020, se presentó el Plan de acción emprendedorismo 2020, de enero de 2013. Este nuevo plan, en línea con las medidas anunciadas anteriormente, se basa en tres ejes: 1) Educar y formar en materia de emprendimiento para promover el crecimiento y la creación de empresas; 2) Crear un entorno en el que los emprendedores puedan desarrollarse y crecer y 3) Establecer modelos y llegar a grupos específicos (COMISIÓN EUROPEA, 2013).

Desde el punto de vista de la instrumentación de la acción pública, cabe destacar lo siguiente. En primer lugar, las políticas de emprendimiento muestran un desplazamiento significativo en el marco de las estrategias analizadas. En sus inicios, a finales de los años noventa, las políticas de desarrollo empresarial apuntaban al apoyo a pequeñas y medianas empresas existentes, a través de beneficios fiscales, líneas de financiamiento, transferencia tecnológica o facilidades administrativas (AUDRETSCH, 2007). Posteriormente, se observa un desplazamiento hacia una política de creación de nuevas empresas, por lo que se priorizan cambios a nivel cultural, educativo o de valores, es decir, de todo aquello que afecta la conducta de los sujetos: "Para que el emprendimiento se convierta en el motor de crecimiento de nuestra economía, Europa necesita una amplia y profunda revolución cultural” (COMISIÓN EUROPEA, 2013: 5). Al implicar este desplazamiento la necesidad de una nueva "mentalidad" o cultura empresarial, estas políticas trascienden el ámbito económico para centrarse en otros aspectos, fundamentalmente educativos.

En segundo lugar, estos instrumentos se inscriben en el paradigma de las llamadas "políticas activas de empleo", es decir, un conjunto de medidas destinadas a favorecer el aumento de la ocupación por medio de servicios de intermediación laboral, incentivos a la contratación y mejoras en la formación de la población activa. Este tipo de medidas se establecen bajo el supuesto de que el problema del desempleo es un problema de "oferta" y dan lugar a un tipo de instrumentos donde la responsabilidad de la creación del empleo recae fundamentalmente en los individuos (SERRANO PASCUAL y CRESPO, 2007).

Por último, cabe destacar que estas políticas proponen instrumentos que resultan problemáticos de cara a los mecanismos de empleo asalariado. Esto se ve reflejado en el solapamiento que se encuentra en los documentos entre "emprendimiento", "autoempleo" y "trabajo autónomo". Por citar un ejemplo: 
[...] el motor de esa recuperación se está fallando: desde 2004, la proporción de personas que prefieren trabajar por cuenta propia antes que convertirse en asalariadas ha disminuido en veintitrés de los veintisiete Estados miembros de la UE. Mientras que el trabajo por cuenta propia era hace tres años la primera opción del $45 \%$ de los europeos, este porcentaje ha bajado ahora hasta el $37 \%$. En cambio, en los Estados Unidos y China esta proporción es mucho mayor: el $51 \%$ y el 56 \% respectivamente" (COMISIÓN EUROPEA, 2013: 4).

Como podemos ver, la Comisión entiende que es un problema que los trabajadores europeos se inclinen hacia el trabajo asalariado en lugar del emprendimiento (entendido como trabajo por cuenta propia) y se propone revertir esa tendencia, sin problematizar las consecuencias que esa transformación pueda tener en los sistemas de bienestar sustentados en el trabajo asalariado.

\section{LA INSTRUMENTACIÓN EN ESPAÑA DE LAS RECOMENDACIONES EUROPEAS}

Como punto central de la instrumentación en España de estas recomendaciones, debe destacarse el alto grado de correspondencia entre la forma y el contenido de las recomendaciones europeas y las medidas adoptadas por el gobierno español. Así, por un lado, encontramos instrumentos que afectan la dimensión cultural, fundamentalmente con políticas educativas y de formación. Por otro, aquellos dirigidos a modificar el "ambiente" en el que se desarrollan las actividades emprendedoras, a través de reformas en la regulación sobre la creación de empresas y la implementación de políticas activas de empleo. A continuación veremos a grandes rasgos cómo se han instrumentado en cada uno de estos campos las políticas de emprendimiento, para concluir posteriormente con una reflexión sobre los efectos de este tipo de instrumentación en el régimen de empleo.

En cuanto al primer eje, cabe destacar que el Estado español ha sido uno de los estados que más ha ajustado su normativa educativa a los requerimientos de la Comisión. En la actualidad, desde la educación primaria hasta la formación superior se contemplan módulos de promoción de los valores y aptitudes del emprendedor. El ejemplo más claro de ello ha sido la inclusión del emprendimiento en la nueva Ley orgánica para la Mejora de la Calidad Educativa, de 2013 (BOE, 2013a), donde se apunta a estimular el espíritu emprendedor de los estudiantes a través una nueva asignatura específica de libre elección llamada Iniciación a la Actividad Emprendedora y Empresarial, que se podría escoger en los cursos de la educación secundaria obligatoria (ESO), dependiendo su implementación de cada Comunidad Autónoma. Estamos en este caso frente a un tipo de instrumento "incitativo", que pretende crear una nueva cultura e incentivar nuevos valores en el mundo del trabajo. 
En lo que refiere al segundo eje, el Programa de Reformas del año 2012 puso de manifiesto el compromiso del gobierno de cara a las instituciones europeas, proponiendo un conjunto de medidas a implementar, como la Estrategia Española de Empleo 2012-2014, donde la promoción de actividades emprendedoras ocupa un lugar relevante, al igual que en la Estrategia de Emprendimiento y Empleo Joven 2013 - 2016. Para dar ejecución a estas estrategias y compromisos se introdujeron novedades en materia legislativa. La instrumentación se divide aquí en dos mecanismos: facilidades a la contratación y promoción del autoempleo.

En cuanto a los primeros, la Ley 3/2012, de 6 de julio, de medidas urgentes para la reforma del mercado laboral, contiene medidas como el Contrato de apoyo a Emprendedores, que procura facilitar la contratación a partir de deducciones fiscales y bonificaciones en la cuota empresarial a la Seguridad Social. En cuanto al fomento del empleo asalariado de los jóvenes, la Ley continúa la línea de promoción del sistema de bonificaciones y reducciones de cuotas empresariales a la Seguridad Social, a la vez que permite considerar la primera experiencia laboral como "causa" justificadora de contratos breves (contrato de "primer empleo joven").

El segundo mecanismo se enmarca en la línea de políticas abierta en el artículo 24.1 c) de la Ley de Empleo 56/2003, donde se establece que el "autoempleo" y la "iniciativa emprendedora" constituyen ámbitos específicos de las políticas activas de empleo, por lo que dentro de las políticas del mercado de trabajo se contemplan las acciones y medidas dirigidas al fomento de las iniciativas empresariales mediante el trabajo autónomo y la economía social (BOE, 2003).

Esta doble línea política de apoyo al emprendimiento quedó plasmada al aprobarse una nueva ley, la 14/2013, de apoyo a los emprendedores y su internacionalización, del el 27 de septiembre de 2013 (BOE, 2013b). En su preámbulo, esta ley establece que:

\footnotetext{
Las causas [del desempleo juvenil] hay que buscarlas, además de en algunas deficiencias que han venido caracterizando a nuestro modelo de relaciones laborales, en la ausencia de una mayor iniciativa emprendedora entre los más jóvenes que haya llevado, ante la falta de oportunidades de trabajo por cuenta ajena, a unos mayores niveles de autoempleo capaces, a su vez, de generar más empleo. Para invertir esta situación, es necesario un cambio de mentalidad en el que la sociedad valore más la actividad emprendedora y la asunción de riesgos. La piedra angular para que este cambio tenga lugar es, sin duda, el sistema educativo. En segundo lugar, el entorno normativo e institucional en el que se desenvuelven las actividades empresariales... (BOE, 2013b: 5. El subrayado es nuestro).
}

Como queda de manifiesto, ambos instrumentos legales coinciden con las recomendaciones de la Comisión Europea citadas anteriormente: por un lado proponen 
medidas que apuntan a un cambio cultural y educativo; por otro, reformas en el entorno normativo. Como veremos, los mecanismos propuestos por la ley tienden a favorecer el autoempleo y fundamentalmente el de carácter individual, a través de determinados instrumentos específicos: el Emprendedor de Responsabilidad Limitada (ERL), como nuevo sujeto jurídico; e instrumentos fiscales de promoción del trabajo autónomo. El siguiente cuadro resume los principales instrumentos adoptados.

Cuadro 2 - Recomendaciones europeas e instrumentación en España de las recomendaciones europeas de emprendimiento.

\begin{tabular}{|c|c|}
\hline Tipo de instrumento & Recomendaciones Europeas sobre emprendimiento \\
\hline $\begin{array}{l}\text { Convencional e } \\
\text { incitativo (soft law) }\end{array}$ & $\begin{array}{c}\text { Método abierto de coordinación } \\
\text { Planes sobre emprendimiento (1998 / } 2004 \text { / 2013). } \\
\text { Programas Nacionales de Reformas (2010-2013) }\end{array}$ \\
\hline Tipo de instrumento & Instrumentación en España de la política analizada \\
\hline $\begin{array}{l}\text { Legislativo y } \\
\text { reglamentario }\end{array}$ & $\begin{array}{l}\text { - Emprendedor de Responsabilidad Limitada (ERL). } \\
\text { - Sociedad Limitada de Formación Sucesiva (SLFS). } \\
\text { - Educación emprendedora en todos los ciclos de formación. } \\
\text { - Contrato de apoyo a Emprendedores. }\end{array}$ \\
\hline Económico y fiscal & $\begin{array}{l}\text { - Incentivos fiscales para nuevos emprendedores. } \\
\text { - "Tarifa plana” en la cuota de autónomos durante } 6 \text { meses. } \\
\text { - Incentivos fiscales a financiadores de proyectos emprendedores. }\end{array}$ \\
\hline $\begin{array}{l}\text { Convencional e } \\
\text { incitativo }\end{array}$ & $\begin{array}{l}\text { - Acuerdos políticos para implementar las medidas sobre emprendimiento en } \\
\text { todas las Comunidades Autónomas. }\end{array}$ \\
\hline $\begin{array}{l}\text { Normas y estándares } \\
\text { de "Buenas } \\
\text { prácticas". }\end{array}$ & $\begin{array}{l}\text { - Educación emprendedora: buenas prácticas en la universidad española } \\
\text { (MIET, 2012) }\end{array}$ \\
\hline
\end{tabular}

Fuente: elaboración propia.

La perspectiva de los instrumentos de acción pública permite en este caso analizar el grado de ajuste o desajuste entre las recomendaciones supranacionales y las medidas tomadas a nivel del Estado español. En este caso, encontramos un alto grado de correspondencia en los dos ejes sugeridos por Europa (reformas culturales/educativas y modificación administrativa y reglamentaria). Además, como veremos en lo que sigue, las medidas adoptadas en España materializan la tendencia presente en las recomendaciones europeas que dirigen el emprendimiento hacia el trabajo autónomo y el autoempleo. 


\section{POSIBLES EFECTOS O TENDENCIAS DE LA INSTRUMENTACIÓN}

En este apartado nos referiremos a los posibles efectos de la instrumentación. Aclaramos la condición de efectos "posibles" o tendencias, porque dado el corto periodo de tiempo transcurrido desde su lanzamiento, es muy pronto para evaluar el impacto efectivo de las medidas en los índices de empleo. Aun así, creemos que es posible indagar la propia lógica y el sentido que encarnan estos instrumentos, en función a los programas de gobierno que los sostienen. En este sentido, destacaremos dos ejes vinculados a las medidas analizadas, que afectan directamente al estatuto salarial, con efectos de remercantilización del trabajo y sus consecuentes efectos sobre las instituciones de bienestar. Estos ejes son: una "huida del derecho del trabajo" y la preeminencia del emprendimiento como autoempleo individual.

\subsection{Huida del derecho del trabajo}

Desde el punto de vista del derecho, las figuras de apoyo a la actividad emprendedora propuestas por la Ley 14/2013 son de carácter marcadamente mercantil: para personas físicas, se crea el "Emprendedor de Responsabilidad Limitada" (ERL) (Capítulo II del Título I), que crea un régimen de limitación de responsabilidad sin adquirir personería jurídica diferenciada de la persona física que realiza la actividad económica o profesional. Como persona jurídica, la "Sociedad Limitada de Formación Sucesiva" (SLFS), que facilita la creación de sociedades sin la necesidad de desembolsos iniciales (Capítulo III del Título I) ${ }^{5}$.

Si se contemplan entonces los mecanismos contractuales ofrecidos por la regulación, nos encontramos con una huida del derecho laboral hacia el autoempleo investido jurídicamente como "actividad emprendedora-sujeto emprendedor". Este instrumento comporta un desplazamiento desde los contratos de trabajo hacia los contratos de servicios civiles o mercantiles $-\mathrm{y}$ a su vez del recibo salarial a la factura por servicios-, lo cual conduce a que las cotizaciones de los empleadores se desplazan hacia "cuotas de autónomos". Como es sabido, este tipo de relaciones laborales implican que es el trabajador quien debe asumir la mayor parte de los riesgos del mercado de trabajo y la economía.

\footnotetext{
5 A su vez, en el ámbito educativo se propone una "herramienta pedagógica", la "miniempresa o empresa de estudiantes" (Disposición adicional novena).
} 
Otro tipo de medidas propuestas por la ley para conseguir la creación de empresas son las simplificaciones del entorno normativo e institucional y el marco jurídico regulatorio. Se propone así la simplificación y liberación de autorizaciones, requisitos y cargas administrativas, alguna de las cuales aporta el Derecho del Trabajo. En este punto, como destaca la especialista en Derecho del Trabajo y expresidenta del Tribunal Constitucional, Emilia Casas Baamonde, el efecto conjunto de ambas leyes de favorecer la actividad empresarial y el trabajo autónomo en nombre del emprendimiento, limita la protección de los trabajadores asalariados en nombre del empleo (LEY 11/2013), o bien intenta favorecer dicha actividad liberándola de las cargas laborales en nombre del emprendimiento (LEY 14/2013). Esto implica, continúa la especialista, conducir a los Derechos del Trabajo y de la Seguridad Social

[...] hacia nuevos objetos fuera de su finalidad de protección de los trabajadores y de equilibrio con los poderes empresariales en las relaciones laborales. Los mecanismos de los Derechos [...] desaparecen en lógicas que obedecen a su sustitución por la atención a los derechos y obligaciones empresariales" (CASAS BAAMONDE, 2013a: 146-147).

Los más claros ejemplos de este último punto son las subvenciones a la contratación, las regulaciones de la reestructuración y reorganización empresarial por razones económicas, técnicas, organizativas o de producción; y las reducciones y bonificaciones de cuotas a la Seguridad Social, todo lo cual conlleva una suerte de "exteriorización" de los trabajadores del objeto de atención de sus normas propias. Incluso el "entorno normativo e institucional" al que la ley apunta, desplaza los centros de gravedad "clásicos" constituidos por la empresa y las relaciones de trabajo. La ruptura del equilibrio entre empresarios y trabajadores que todo ello conlleva implica una re-mercantilización de las relaciones laborales. Es decir, dejar al trabajo a merced de los ciclos del mercado.

Si bien algunos nuevos emprendedores revelan un verdadero proceso, aunque muchas veces forzado, de transición de "trabajador" a "empresario", un importante número de ellos pasan a engrosar las filas de los llamados "falsos autónomos", es decir, a trabajadores que están inscriptos en el Régimen Especial de Trabajadores Autónomos (RETA) pero que de hecho trabajan en relación de dependencia (VALLECILLO y MOLINA NAVARRETE, 2013). Según datos del diario El País, entre 2012 y principios de 2015 se detectaron 2.372 "falsos autónomos" (EL PAÍS, 2015).

De este modo, se desvanece la figura del trabajador asalariado "clásico", debido a la emergencia de estatutos de promoción del trabajo en los que el trabajador adquiere cualidades de "empresario", dificultando de esa forma la identificación de los sujetos laborales y 
empresariales. Así, las formas jurídicas favorecen una primacía del autoempleo individual y aparecen una multiplicidad de efectos de desplazamiento de las normas laborales hacia regímenes especiales más "liberales y baratos" (MOLINA NAVARRETE, 2013: 189). El despido libre, la responsabilización de los propios trabajadores de pagar sus cuota de autónomos y la mayor incertidumbre en el empleo que estas figuras comportan, permiten hablar de una remercantilización del trabajo, en cuanto se borran las garantías que el Derecho del Trabajo y de la Seguridad Social ofrecían y tienden a hacer del trabajo una mercancía más entre otras.

Por ello, lo que se promete en la ley como un salto cualitativo en el mundo del trabajo, podría terminar alimentando viejos problemas, como el de las "zonas grises", es decir, las zonas en que concurren diversas formas de prestación de servicios retribuidos, mercantiles y laborales, y las múltiples estrategias de huida de la regulación sociolaboral basada en el Derecho del Trabajo (CASAS BAAMODE, et al. 2013b).

\subsection{Preeminencia del autoempleo individual y por necesidad}

Diversos estudios en el campo del Derecho del trabajo (CASAS BAAMONDE, 2013a; VALLECILLO y MOLINA NAVARRETE, 2013) han destacado dificultades que emergen a raíz de la multiplicación que los programas y leyes hacen de las formas subjetivas o personificadas de la organización jurídico-económica de la actividad de emprender: emprendedor (individual o colectivo), autónomos, autoempleo. Esto supone cierta dificultad a la hora de distinguir trabajadores autónomos, auto-empleados y emprendedores. Aun así, lo cierto es que desde el punto de vista de las estadísticas sobre emprendimiento, el Estado Español, siguiendo la línea de las instituciones europeas y del Global Entrepreneurship Monitor, contempla como emprendedores todas las altas de sociedades mercantiles realizadas en un año, independientemente de su tamaño o actividad.

No se trata de negar que haya contempladas en la ley medidas auténticamente dirigidas al fortalecimiento del emprendimiento, como la financiación o el apoyo técnico. Pero, de hecho, a pesar de la creación de la figura de la "Sociedad Limitada de Formación

\footnotetext{
${ }^{6}$ El Global Entrepreneurship Monitor (GEM) constituye la más importante red mundial sobre emprendimiento y tiene por objetivo hacer que la información de alta calidad sobre la iniciativa emprendedora esté disponible para el mayor público posible. http://www.gem-spain.com/ [Visitado el 10/07/2015].
} 
sucesiva", no han tenido especial acogida en la Ley la promoción de formas de emprendimiento como el "emprendimiento social" o "autoempleo colectivo". Como ejemplo de ello, quienes han optado por una forma societaria no económico-social (cooperativas de trabajo asociado) y no por la actividad de autónomos e régimen de personas física, no se han visto beneficiados de la misma forma en las reducciones de la Seguridad Social ${ }^{7}$.

Si bien la SLFS puede entenderse como una figura más apropiada para la actividad emprendedora, la instrumentación de una de las principales medidas adoptadas por la Ley, la tarifa social plana (reducción de aportes a la seguridad social durante el primer año), hace que la actividad "emprendedora" quede ligada fundamentalmente al trabajo autónomo individual y, en menor medida, al autoempleo colectivo o social (cooperativas de trabajo asociado, sociedades laborales). Por ejemplo, las bonificaciones dejan de corresponder si se produce la contratación de trabajadores por cuenta ajena. Por ello, es válido preguntarse si la instrumentación de la "tarifa social plana" favorece a los emprendedores, o a los "falsos autónomos", tal como se describió hace un momento.

En efecto, entre el mes de marzo de 2013, cuando entró en vigor la tarifa plana, y julio del mismo año, se dieron de alta unas 50.000 nuevas altas, al tiempo que disminuía en 38.000 el Régimen Especial de Trabajadores Autónomos. Como destaca Molina Navarrete (2013), parece dudoso que 50.000 jóvenes hayan logrado crear una mínima estructura empresarial. Todo indicaría, en cambio, que se ha producido un "efecto desplazamiento" entre los distintos regímenes de trabajadores autónomos.

Por otra parte, el propio preámbulo de la Ley 14/2013 reconoce que la "falta de oportunidades de empleo" asalariado, está llevando a muchas personas a adoptar una actividad "emprendedora" (BOE, 2013b: 2). El problema es que esta "opción” no viene en la mayor parte de los casos motivada por una oportunidad de negocios, con su plan de inversiones y su estrategia de crecimiento a mediano y largo plazo, sino como una necesidad de hacer frente al desempleo por medio del autoempleo (MOLINA NAVARRETE, 2013).

\footnotetext{
7 Molina Navarrete (2013: 196) destaca que esta primacía del autoempleo individual por sobre las formas de trabajo cooperativo despertó quejas y críticas en el Congreso de los Diputados por parte del Parlamentario del Grupo Mixto Tardá i Coma, al cual cita: "Señorías, debo decirles que intervenimos con una cierta decepción ante este proyecto de ley, que desde hoy será ya una ley aprobada por el conjunto de las Cámaras. Y digo que es una decepción porque el Grupo Parlamentario Popular, el grupo mayoritario, había adquirido el compromiso de incorporar la economía social a este proyecto. Estamos viviendo una decepción, que es extensible a todo el sector de la economía social, que había depositado en esta ley muchas esperanzas". http://www.congreso.es/portal/page/portal/Congreso/PopUpCGI?CMD=VERLST\&BASE=pu10\&DOC $\mathrm{S}=1-1 \& Q \mathrm{Q} E \mathrm{RY}=(\mathrm{DSCD}-10-\mathrm{PL}-139 . \mathrm{CODI}$.) [Visitado el 04-07-2014].
} 
De allí que primen las empresas sin asalariados y, en menor medida, el autoempleo colectivo o social. De hecho, como puede apreciarse en el Gráfico 1, de las 343.491 empresas dadas de alta al 1 de enero de 2014, un 75\% lo hizo sin empleados y un 20\% surgió con entre 1 y 5 empleados.

Gráfico 1 - Empresas creadas en España al 1 de enero de 2014

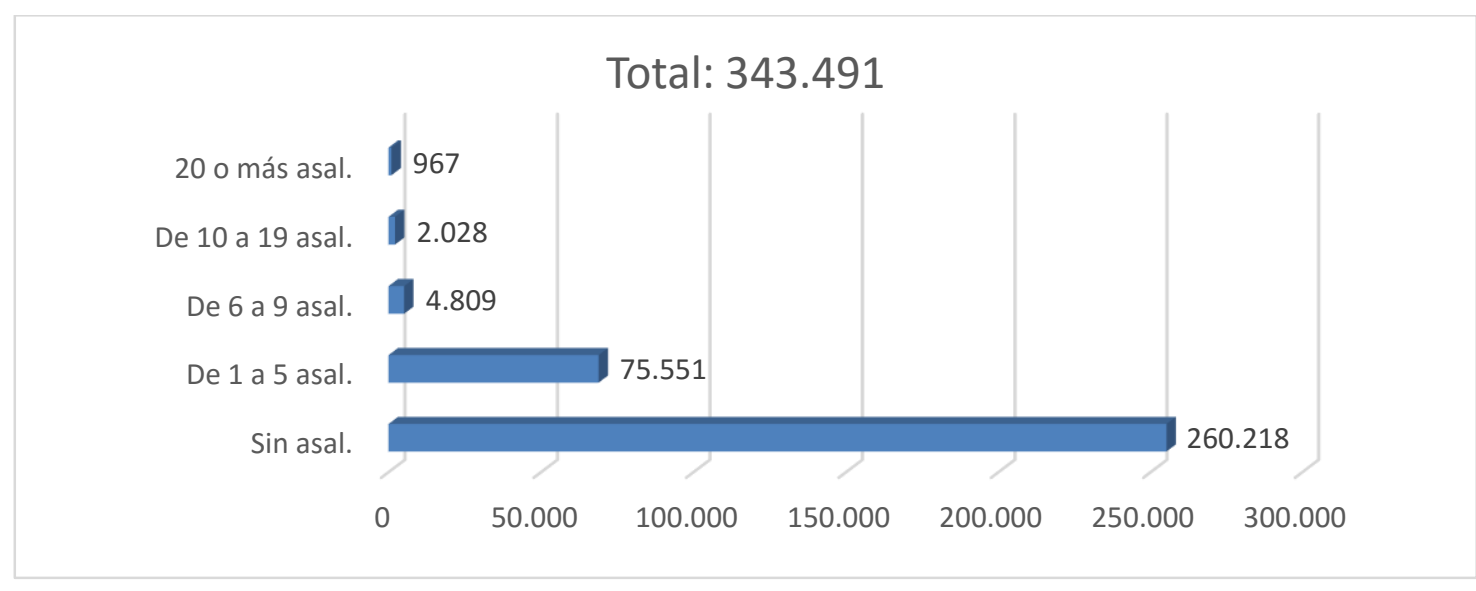

Fuente: elaboración propia a partir de INE.

En el cuadro 3 se exponen los datos del GEM, que miden la distribución de los emprendedores en fase inicial en España, por el tamaño en empleo de sus proyectos de negocios en fase inicial (de 0 a 42 meses). Esta medición, a diferencia de la presentada anteriormente, permite ver la evolución de los primeros años de los emprendimientos. Allí puede verse que los nuevos emprendedores sin empleados representan el 54,7\% del total para el año 2014, mientras que los nuevos emprendedores con entre 1 y 5 empleados representan el 37,1\%. Si bien estos datos matizan los anteriores, ponen también de manifiesto la tendencia de la preeminencia de un tipo de emprendedor individual y sin asalariados.

Cuadro 3 - Distribución de los emprendedores en fase inicial en España, por el tamaño en empleo de sus proyectos de negocios en fase inicial (de 0 a 42 meses)

\begin{tabular}{|c|c|c|c|c|}
\hline Año & Sin empleados & $\mathbf{1 - 5}$ empleados & $\mathbf{6 - 1 9} \mathbf{~ e m p . ~}$ & $\mathbf{2 0}$ y más emp. \\
\hline $\mathbf{2 0 1 2}$ & $57,10 \%$ & $36,40 \%$ & $5,70 \%$ & $0,80 \%$ \\
\hline $\mathbf{2 0 1 3}$ & $52,20 \%$ & $39,56 \%$ & $5,09 \%$ & $3,15 \%$ \\
\hline $\mathbf{2 0 1 4}$ & $54,7 \%$ & $37,1 \%$ & $6,5 \%$ & $1,7 \%$ \\
\hline
\end{tabular}

Fuente: elaboración propia a partir de GEM (2014). 
A ello hay debe sumarse que, si se tiene en cuenta que la mayor parte de los "emprendedores" corresponden al tipo "por necesidad" (GEM, 2013), puede establecerse como hipótesis que buena parte de los casos que ese nuevo marco regulador presenta como "emprendimiento", responden al comportamiento de "empleo refugio", típico en períodos de crisis (VALLECILLO Y MOLINA NAVARRETE, 2013).

Por otra parte, más allá de la declaración de intenciones del legislador, el análisis de las medidas de la Ley 14/2013, pone de manifiesto que estimulan el autoempleo en su primera fase, sin generar nuevos estímulos para que posteriormente contrate nuevos empleados. De hecho, en los artículos 29 y 30, las reducciones a las cuotas de la Seguridad Social se limitan a los autónomos que no tengan trabajadores por cuenta ajena a su cargo (Casas Baamonde, 2013a: 140).

Cabe recordar que tanto las recomendaciones de las instituciones europeas como la propia legislación española no se limitan a cambios en el marco regulador del mundo del trabajo, sino que apuntan a un cambio cultural. Esta última dimensión es difícil de ser medida o cuantificada, más aún si se tiene en cuenta que los cambios culturales ocurren en períodos relativamente largos de tiempo. El instrumento de medición y comparación que suele utilizarse en este punto son los informes del General Entrepreneurship Monitor (GEM), donde se relevan "los valores y percepciones y aptitudes emprendedoras", preguntando a la población adulta activa sobre su "intención" de emprender o su preferencia entre el trabajo por cuenta propia o el trabajo por cuenta ajena (GEM, 2013). Las respuestas negativas a la primera pregunta y la respuesta favorable al trabajo por cuenta ajena en la segunda, son consideradas como un indicador de la "falta de espíritu emprendedor".

Cuadro 4 - percepciones, valores y aptitudes para emprender de la población adulta en 2014. Análisis por tipo de economía.

\begin{tabular}{|c|c|c|c|c|c|c|}
\hline \multicolumn{3}{|c|}{$\begin{array}{c}\text { Percepciones de la población adulta sobre sus valores y } \\
\text { aptitudes para emprender }\end{array}$} & \multicolumn{3}{c|}{$\begin{array}{c}\text { Percepciones de la población } \\
\text { adulta sobre la cultura y su } \\
\text { influencia en el emprendimiento }\end{array}$} \\
\hline $\begin{array}{c}\text { Percepción de } \\
\text { oportunidades [de } \\
\text { negocio] }\end{array}$ & $\begin{array}{c}\text { Conocimientos } \\
\text { y habilidades } \\
\text { para } \\
\text { emprender }\end{array}$ & $\begin{array}{c}\text { Miedo al } \\
\text { fracaso } \\
\text { como } \\
\text { obstáculo } \\
\text { para } \\
\text { emprender }\end{array}$ & $\begin{array}{c}\text { Modelos } \\
\text { de } \\
\text { referencia }\end{array}$ & $\begin{array}{c}\text { Equidad } \\
\text { en los } \\
\text { estándares } \\
\text { de vida de } \\
\text { la } \\
\text { sociedad }\end{array}$ & $\begin{array}{c}\text { Emprender } \\
\text { como } \\
\text { buena } \\
\text { opción } \\
\text { profesional }\end{array}$ & $\begin{array}{c}\text { Emprender } \\
\text { brinda } \\
\text { estatus } \\
\text { social y } \\
\text { económico }\end{array}$ \\
\hline
\end{tabular}

\footnotetext{
${ }^{8}$ El GEM establece una distinción entre emprendedores por oportunidad y emprendedores por necesidad. El primero, se considera tal cuando se emprendió para aprovechar una oportunidad de negocio, el segundo, cuando se inició un negocio porque no había mejores alternativas de empleo.
} 


\begin{tabular}{|c|c|c|c|c|c|c|c|}
\hline España & 22,6 & 48,1 & 46,5 & 35,7 & 71,9 & 53,9 & 49,0 \\
\hline $\begin{array}{c}\text { Media } \\
\text { UE/ } \\
\text { EEUU }\end{array}$ & 88,9 & 42,0 & 42,1 & 31,6 & 62,5 & 55,1 & 68,2 \\
\hline
\end{tabular}

Fuente: GEM España (2014).

Más allá del análisis de las magnitudes de cada una de estas variables, nos interesa destacar lo siguiente: los instrumentos de formación en emprendimiento apuntan a modificar la cultura emprendedora, de modo que las magnitudes para España se acerquen tanto como sea posible a la media; esto es, que la población adquiera una cultura más afín al emprendimiento. Esto queda de manifiesto cuando el informe agrega al análisis de los datos de la tabla, la siguiente reflexión:

\footnotetext{
Se destaca el camino que aún queda por recorrer en el reforzamiento permanente de los valores, percepciones y aptitudes de la población hacia el emprendimiento no solo como generación de empresas/empleo sino como una forma de vida que se puede ejercer en cada actividad que se decida acometer. Todo ello afecta a la evolución de nuestra cultura emprendedora y a la formación como instrumento para sensibilizar, educar y capacitar a las personas que desean emprender (GEM, 2014: 34).
}

A la luz de lo expuesto anteriormente, la solicitada metamorfosis de la cultura emprendedora, tiene un impacto relevante en las relaciones laborales al conjugarse con las declaraciones de intención del legislador y los instrumentos jurídicos propuestos. Por un lado, tenemos el ya mencionado solapamiento entre "emprendimiento", "autoempleo" y "trabajo autónomo" y, por otro, el vínculo entre la promoción de la cultura emprendedora y la búsqueda de empleos alternativos al asalariado, incitando de esta forma la fuga del contrato de trabajo hacia formas mercantiles de empleo (MOLINA NAVARRETE, 2013). Se asiste de esta forma a un intento de metamorfosis de (llamada reconversión o reciclaje) de empleados y en su mayor parte de desempleados en "emprendedores-autoempleados" (KAUTONEN, 2010).

Pero, como destacan Congregado (2006), debe tenerse en cuenta que el autoempleo no puede considerarse necesariamente como estado final, ya que los determinantes de transición al autoempleo pueden variar según sea el estado final el de autoempleo o el de empleador con asalariados. Por tanto, las políticas de promoción de la actividad emprendedora no deberían contentarse con la transición de desempleados o asalariados hacia el emprendimiento individual, sino apuntar al sostenimiento y crecimiento de esas empresas. 


\section{A MODO DE CONCLUSIÓN}

Como hemos visto, la perspectiva de los Instrumentos de Acción Pública permite arrojar luz sobre la forma en que los programas de gobierno, en este caso a nivel supranacional, son instrumentados a nivel estatal con determinados efectos sobre su campo de aplicación. A partir de ello, es posible identificar las estrategias de las políticas públicas en su objetivo de estructurar los comportamientos individuales y colectivos. En el caso que nos ha ocupado, hemos visto cómo el Estado español ha instrumentado las políticas de emprendimiento siguiendo estrictamente las propuestas de las instituciones europeas, lo cual ha dado lugar a un tipo de mecanismo que tiende a superponer la figura del emprendedor a la modalidad de autoempleo por necesidad.

Hemos puesto de manifiesto que las políticas de emprendimiento en España se han plasmado en un conjunto de instrumentos de legislación laboral (figuras jurídicas), de tipo fiscal (bonificaciones), e incitativo (cambio cultural e incentivo a devenir emprendedor) y que a su vez se basan en macroinstrumentos vinculados a normas y estándares de buenas prácticas, a partir de los cuales se instrumentan y evalúan las medidas adoptadas entre los Estados miembros. Todo ello tiene como efecto que el tipo de empleo creado sea mayoritariamente auto-empleo, lo cual conduce a un desplazamiento de los mecanismos del derecho del trabajo que sustentan a la figura del trabajo asalariado (central en los regímenes de bienestar europeos) y favorece el establecimiento de regímenes de empleo liberales, donde el trabajo depende estrechamente de los mecanismos de mercado.

Pero las formas de instrumentación de las políticas de promoción del emprendimiento son tema de permanente debate académico y político. La vía recomendada por las instituciones europeas ha sido criticada por diversos autores, que sostienen que este tipo de políticas puede adquirir otra fisonomía y conducir a la creación de empleo de calidad (SHANE, 2009). Es decir, los instrumentos elegidos por el gobierno español en el periodo 2010-2013 para instrumental las recomendaciones europeas, podrían haber adquirido otras características distinta a las actuales.

De esta forma, el análisis de la modalidad de instrumentación de las políticas sociales contribuirá a los debates en tono al rol de Europa en la política doméstica, tanto a nivel académico como del propio proceso de las políticas públicas. Si, según esta perspectiva, se intenta poner de manifiesto que la fisonomía que adoptan los instrumentos no reviste carácter "necesario", nos enfrentamos al debate teórico-político sobre la posibilidad de cambiar la forma en que se instrumentan las políticas sociales y se organiza lo social. Así, lo que este artículo ha pretendido aportar a la discusión sobre las políticas sociales en España, 
es que incluso si se asume que la innovación, la creatividad, la asunción de riesgos, etc. son características necesarias en la economía actual, pueden pensarse formas distintas de instrumentación de las políticas de emprendimiento que no se orienten fundamentalmente hacia formas mercantiles de creación de empleo centradas en el individuo.

Por otra parte, esa posible diversidad de modalidades de instrumentación debe ser motivo para el desarrollo de estudios comparativos a nivel europeo e internacional, que pongan de manifiestos las distintas formas de instrumentación que un programa de gobierno puede adquirir. En este sentido, la perspectiva de los IAP permitiría indagar los niveles de correspondencia entre las recomendaciones europeas y las medidas adoptadas en cada uno de los países de la Unión, para pensar formas de instrumentación distintas a las existentes.

\section{REFERÊNCIAS}

ALONSO, L. (2007). La Crisis de la ciudadanía laboral. Rubí, Anthropos.

AUDRETSCH, D. B. (2007). The entrepreneurial society. Oxford, Oxford University Press.

BOE (2003) Ley 56/2003, de 16 de diciembre, de Empleo. Disponible en: https://www.boe.es/buscar/pdf/2003/BOE-A-2003-23102-consolidado.pdf [Visitado el $1 / 7 / 2015]$.

BOE (2013a) Ley 11/2013, de 26 de julio, de medidas de apoyo al emprendedor y de estímulo del crecimiento $y$ de la creación de empleo. Disponible en http://www.boe.es/diario_boe/txt.php?id=BOE-A-2013-8187 [Visitado el 02-07-2014].

BOE (2013b) Ley 14/2013, de 27 de septiembre, de apoyo a los emprendedores y su internacionalización. Disponible en http://www.boe.es/diario_boe/txt.php?id=BOE-A-201310074 [Visitado el 02-07-2014].

BRUNO, I. (2006). Déchiffrer l'Europe compétitive. Étude du benchmarking comme technique de coordination intergouvernementale dans le cadre de la stratégie de Lisbonne, Thèse de doctorat en science politique, IEP Paris.

CASAS BAAMONDE, M. E. (2013a). El derecho del trabajo y el empleo asalariado en los márgenes: De nuevo el emprendimiento y el autoempleo. Relaciones Laborales: Revista Crítica De Teoría y Práctica, No 11, pp. 137-164.

CASAS BAAMONDE, M. E; RODRIGUEZ PIÑERO, M. y VALDÈS DAL-RÉ, F. (2013b) La huida del derecho del trabajo hacia el "emprendimiento", las reformas de la Reforma Laboral y otras reformas: la L 11/2013 y el RDL 11/2013. Relaciones Laborales: Revista Crítica De Teoría y Práctica, No 10, pp. 1-29.

COMISIÓN EUROPEA (1998) Fomento del espiritu de empresa en Europa: prioridades para el futuro. Bruselas, Comisión Europea. 
COMISIÓN EUROPEA (2001) La gobernanza Europea. Un libro blanco. Bruselas, Comisión Europea.

COMISIÓN EUROPEA (2001) El programa europeo en favor del espiritu empresarial. Bruselas, Comisión Europea.

COMISIÓN EUROPEA (2013) Plan de acción sobre emprendimiento 2020. Relanzar el espiritu emprendedor en Europa, Bruselas, Comisión Europea.

CONGREGADO, E; GOLPE, A. y MILLÁN, J. (2006) Los autónomos en el marcado de trabajo español: situación, políticas y retos. Perspectivas del sistema financiero. $\mathrm{N}^{\circ}$ 86, pp. 1-20.

CONSEJO EUROPEO (2000) Conclusiones de la presidencia. Consejo Europeo de Lisboa. 23 y 24de marzo de 2000. Luxemburgo, Oficina de Publicaciones de la Unión Europea.

DEHOUSSE, R. (2003), The Open Method of Coordination: a New Policy Paradigm?. Les Cabiers européens de Sciences Po, No 3, pp. 1-30.

EL PAÍS (2015) Empleo detecta a 2.372 falsos autónomos desde 2012. En línea: http://economia.elpais.com/economia/2015/05/17/actualidad/1431895703_777581.html [Visitado el 26/06/2015].

GEM (2013) Informe GEM España 2013. Red Española de Equipos Regionales.

GEM (2014) Informe GEM España 2014. Red Española de Equipos Regionales.

GOBIERNO de ESPAÑA (2013) Programa Nacional de reformas del Reino de España. En línea: http://www.empleo.gob.es/es/sec_trabajo/debes_saber/pnr/PNREspanya2013.pdf [Visitado el 11 de julio de 2015].

HALPERN, C., LASCOUMES, P. y LE GALES, P. (2014). Introduction. L'instrumentation et ses effets débats et mises en perspective théoriques. En HALPERN, C; LASCOUMES, P. y LE GALES, P. (Eds.), L'instrumentation de l'action publique. Paris: Presses de Science Po.

HOOD, Ch. (2007) Intellectual Obsolescence and Intellectual Makeovers: Reflections on the Tools of Government after Two Decades. Governance, Vol. 20, No 1, pp. 127-144.

KAUTONEN, T; DOWN,S; WELTER, F. et al. (2010) 'Involuntary self-employment' as a public policy issue: a cross-country European Review. International Journal of Entrepreneurial Behaviour \& Research. Vol. 16, No 2, pp. 112-129.

LABORIER, P. y LASCOUMES, P. (2005), "L'action publique comprise comme gouvernementalisation de l'Etat'. En MEYET, S; NAVES, M.-C. y RIBEMONT, T. (dir.) Travailler avec Michel Foucault. Retours sur le politique, Paris, L'Harmattan, pp. 37-62.

LASCOUMES P. y LE GALÈS P. (dir.), Gouvernerpar les instruments, Paris, Presses de Sciences Po, 2004.

LASCOUMES, P. y SIMARD, L. (2011) L'action publique au prisme de ses instruments, Revue Française de Science Politique, Vol. 61, No 1, pp. 5 - 22.

MESS (Ministerio de Empleo y Seguridad Social de España) (2013) Programa Nacional de Reformas 2013. 
MIET (2012). Educación Emprendedora: buenas prácticas en la universidad española. Madrid: Ministerio de Industria, Energía y Turismo de España.

MOLINA NAVARRETE, C. (2013). La dimensión socio-laboral del pretendido -¿o pretencioso?- nuevo "estatuto promocional del emprendedor". Estudios Financieros. Revista De Contabilidady Tributación: Comentarios, Casos Prácticos, No 369, pp. 177-256.

MONEREO PÉREZ, J. L. (2013). “Introducción”. En Molina Navarrete C. y Moreno Vidal, M. (Eds.), Manual de derecho del trabajo (11 ${ }^{\mathrm{a}}$ ed. ed.). Granada, Comares.

PRIETO, C. (2013) From flexicurity to social employment regimes. En KEUNE, M. y SERRANO PASCUAL, A. Deconstructing flexicurity: alternative approaches, New York, Routledge.

ROTH, A. (2008), Perspectivas teóricas para el análisis de las políticas públicas: ¿de la razón científica al arte retórico?. Estudios Políticos, No 33, pp. 67-91.

SERRANO PASCUAL, A. y CRESPO, E. (2007) The government of activation policies by EU institutions. International Journal of Sociology and Social Policy, Vol. 27, No 9/10, pp. 376-386.

SHANE, S. (2009) Why encouraging more people to become entrepreneurs is bad public policy. Small Business Economics, Vol. 33, No 2, pp. 141-149.

VALLECILLO GÁMEZ, M. R., y MOLINA NAVARRETE, C. (2013). La nueva ley de fomento del autoempleo: en busca de "el dorado". Estudios Financieros. Revista De Trabajo y Seguridad Social: Comentarios, Casos Prácticos: Recursos Humanos, No 367, pp. 55-102. 